\title{
From insulin detemir to glargine: Effect on glycemic control and psychological wellbeing in patients with diabetes mellitus type 2 in daily practice
}

\author{
Thiemo F. Veneman ${ }^{1 *}$, Fred E. M. G. Storms ${ }^{2}$, Ingo A. Eland ${ }^{3}$, Paul K. P. Bouter ${ }^{4}$ \\ ${ }^{1}$ Department of Internal Medicine, Ziekenhuisgroep Twente, Almelo, Netherlands; ${ }^{*}$ Corresponding Author: tfveneman@hetnet.nl \\ ${ }^{2}$ Department of Internal Medicine, Sint Antoniusziekenhuis, Utrecht, Netherlands \\ ${ }^{3}$ Department of Internal Medicine, Sint Antoniusziekenhuis, Nieuwegein, Netherlands \\ ${ }^{4}$ Department of Internal Medicine, Jeroen Bosch Ziekenhuis, 's-Hertogenbosch, Netherlands
}

Received 29 November 2011; revised 31 December 2011; accepted 17 January 2012

\section{ABSTRACT}

The success of a specific treatment is traditionally judged according to parameters such as HbA1c. However, other, patient-reported outcomes (PRO) of (insulin) therapy, become increasingly more important. The introduction of (basal) insulin-analogues could possibly improve PRO, particularly "quality of life". Direct comparative studies between once daily insulin glargine and once to twice or twice daily insulin detemir have previously shown differences in insulin dosage, dosing frequency and weight gain. Whether this leads to a difference in quality of life in patients who are transferred from insulin detemir to insulin glargine remains to be determined. To establish the effect of insulin glargine on quality of life and patient satisfaction in patients with DM2 who are in poor metabolic control with a (human) basal insulin, a large prospective, observational study in Dutch daily practice was performed. The results of the patient population switched from NPH-insulin to insulin glargine have been published previously. In this article the results of the group of patients treated with insulin detemir before the observation period are described. The results of this observational study show that changing basal insulin therapy to insulin glargine in patients with DM2 who are in poor glycemic control with insulin detemir leads to a clinically significant improvement of glycemic control as well as emotional wellbeing, despite a small increase in weight. Whether other factors such as decreased dosing frequency play a role remains to be determined by future studies.

"Disclosures: All authors are members of the national advisory board of sanofi-aventis Netherlands.
Keywords: Insulin Glargine; Insulin Detemir; Psychological Wellbeing; Metabolic Control; Type 2 Diabetes

\section{INTRODUCTION}

Diabetes mellitus type 2 (DM2) is characterized by insulin resistance and a progressive decline of the insulin secretory capacity. This pathophysiological mechanism necessitates intensification of therapy in time for most patients [1]. As a consequence the majority of patients with DM2 become dependent on treatment with insulin, with our without the combination with oral glucose lowering medication [2]. It is important to realize that good glycemic control, early in the disease process, is needed to prevent long-term (cardio) vascular complications [3]. Different guidelines, such as the ADA/EASD consensus statement and the Dutch (NHG) standard Diabetes Mellitus type 2 , are focused on timely intensification of treatment $[4,5]$. If good glycemic control cannot be reached by oral glucose-lowering medication alone, addition of once daily basal insulin is considered to be the next step. However, several studies show that in many instances this does not occur in daily practice [6,7]. One retrospective cohort analysis based on the THIN (The Health Improvement Network) database showed that $50 \%$ of patients with DM2 who were in insufficient metabolic control on oral glucose lowering medication alone received treatment with insulin not until 4.9 years later [6]. The postponement of insulin therapy is based on negative perceptions regarding insulin in patients as well as in doctors. Among them are supposed complexity of the treatment, worries about wellbeing, fear for weight increase and fear for hypoglycemia [8-14]. It is not fully clear to what extent insulin therapy influences the quality of life and/or patient satisfaction regarding therapy. Apart from studies showing a beneficial effect on these pa- 
rameters [15-18], there are even so studies showing a neutral $[19,20]$ or a negative effect [21-23].

Apart from the traditional parameters such as $\mathrm{HbAlc}$, other, patient reported outcomes (PRO) of (insulin) therapy, become an increasingly more important part in the judgment of the success of a specific treatment [24]. The introduction of (basal) insulin-analogues could possibly improve PRO, particularly with regards to quality of life $[17,18]$. Direct comparative studies between once daily insulin glargine and once to twice or twice daily insulin detemir have previously shown no clinically relevant difference in glycemic control and equal low number of hypoglycemic events. However differences in insulin dosage, dosing frequency and weight gain were observed. Whether this leads to a difference in quality of life in patients who are transferred from insulin detemir to insulin glargine remains to be determined. To establish the effect of insulin glargine on quality of life and patient satisfaction in patients with DM2 who are in poor metabolic control with a (human) basal insulin, a large prospective, observational study in Dutch daily practice was performed [25]. The results of the patient population switched from NPH-insulin to insulin glargine have been published previously [25]. In this article the results of the group of patients treated with insulin detemir before the observation period are described.

\section{METHODS}

\subsection{Patient Selection}

The observational ESPRIT (Effect Study on Patient Reported outcomes in Insulin glargine Treatment) study was performed between 2005 and 2008 [25]. In 116 centers in The Netherlands patients with DM2 on NPH-insulin, mixed insulin or insulin detemir \pm oral glucose lowering medication \pm short acting-insulin were asked to participate in the study by their doctor, after the decision was made that the current basal insulin needed to be replaced by once daily insulin glargine. The decision to start with insulin glargine was taken by the caregiver based on clinical decisionmaking and thus independent of participation in the study. Participation in the study did not interfere with normal daily practice. Patients were asked to fill out a questionnaire on three different occasions which took approximately 15 minutes. Because the study had an observational and non-invasive character approval of an ethical committee was not needed. A total number of 510 patients participated in the study, 83 of them initially treated with insulin detemir. Clinical parameters as well as PRO were measured at baseline and at 3 and 6 months respectively.

\subsection{Measurements}

Demographic and clinical data were obtained by self reporting and consisted of age, gender, body weight, time since diagnosis, use of medication, frequency of hypoglycemia, presence of diabetes related complications as well as co-morbidity. At baseline as well as during the follow-up visits after 3 and 6 months $\mathrm{HbA1c}$ and fasting glucose concentration were measured. Adverse events were recorded and treated accordingly by the physician.

PRO for the establishment of patient well being consisted of three validated questionnaires. The Dutch version of the "Worry subscale" of the "Hypoglycaemia Fear Survey" (HFS-w), a validated 13-item questionnaire, was used to measure worrying about hypoglycemia [26]. To clarify interpretation of the data the HFS-w scores were transformed to a " 0 -to- 100 -scale". The commonly used "Diabetes Symptom Checklist-revised" (DSC-r) was used to measure the severity of diabetes related symptoms. The DSC-r consists of 34 items, grouped in 8 subscales: hyperglycemia, hypoglycemia, cognitive defects, fatigue, cardiovascular problems, neuropatic pain, neuropathic sensitivity and ophthalmologic functioning $[27,28]$. Each item of the DSC-r asks for the presence or absence of specific symptoms (yes/no) and, if present, to which extent on a 5-point Likert scale. Next the scores are transformed to a 0 tot 100 score in order to obtain the DSC-r total score. A similar transformation is applied on different DSC-r subscales. General emotional wellbeing was measured with the WHO-5 wellbeing index [29]. The WHO-5 measures positive mood, vitality and general interest. Item scores are added in order to obtain a total wellbeing-score and transformed to a 0 to 100 scale. Low scores indicate less wellbeing. The WHO-5 questionnaire is a suitable method to screen for depressive mood disorders with a cutoff score of 28 [30].

\subsection{Statistical Analysis}

The primary variable was change of the PRO's: HFS-w, DSC-r, and WHO-5 from baseline (before initiation of treatment with insulin glargine) to 3 and 6 months after initiation of treatment with insulin glargine. This was determined with "time" as an independent dummy variable using the Generalized Estimating Equations (GEE) analysis. Secondary variables (change in $\mathrm{HbA}_{1 \mathrm{c}}$, fasting blood glucose, the number of symptomatic, nocturnal and severe hypoglycemic episodes during the past month and bodyweight after 3 and 6 months) were analyzed in a similar fashion. If variables were not normally distributed they were transformed according to a natural logarithm. This was the case for the number of symptomatic, nocturnal and severe hypoglycemic episodes during the past month, the HFS-w, DSC-r total score and the subscales of the DSC-r. Because of the setting of the study which is performed in daily practice and the lack of controls, observational studies in general are at risk to miss 
data. In order to optimize the use of available data several imputation techniques are available. In this study we choose for the technique of multiple imputation, presently the most robust method to handle missing data [31]. Analyses are performed using STATA, version 10.0 (StataCorp, College Station, TX, USA). P-values $<0.05$ were considered to be statistically significant.

\section{RESULTS}

Baseline demographic data and change in time of clinical parameters are shown in Table 1. Prior to the start of treatment with insulin glargine out of the 510 patients with DM2 from the total ESPRIT cohort 280 patients were treated with NPH-insulin, 147 with mixinsulin and 83 with insulin detemir. In the patients treated with insulin detemir the mean dose of the basal insulin at baseline was $39 \pm 26$ units per day, comparable to the mean dose of insulin glargine at the start of the study ( $39 \pm 22$ units per day). In addition to the basal insulin, patients in the insulin detemir group used other glucose lowering medication: oral medication $(\mathrm{N}=7$, $8 \%)$, other insulin $(\mathrm{N}=56,67 \%)$ or a combination of both $(\mathrm{N}=20,24 \%)$.

The mean $\mathrm{HbAlc}$ at baseline was $8.4 \% \pm 1.1 \%$ and de- creased to $8.0 \pm 1.2 \%$ after 3 months. The decrease was persistent, with an $\mathrm{HbAlc}$ of $8.0 \% \pm 3.1 \%$ at the end of the observation period of 6 months ( $p=0.001$ vs. baseline, Table $\mathbf{1}$ ).

Comparable data were found regarding the decrease of the fasting blood glucose concentration (FBG). The FBG decreased from $10.3 \pm 3.8 \mathrm{mmol} / \mathrm{L}$ at baseline to $8.7 \pm$
$3.0 \mathrm{mmol} / \mathrm{L}$ after 3 months and $8.6 \pm 3.1 \mathrm{mmol} / \mathrm{L}$ after 6 months of follow-up ( $p=0.001$ vs. baseline). The improvement of glycemic control was accompanied by a slight increase of the BMI as well as of the use of the total units of basal insulin (Table 1).

At baseline $66 \%$ of the patients indicated to have had a symptomatic hypoglycemic episode in the 3 months prior to the start of the study. This number does not change significantly during follow-up, 70\% after 6 months $(\mathrm{p}=$ 0.475). At baseline the percentage of patients with 1 or more nocturnal hypoglycemic episodes in the 3 months prior to the start of the study was $37 \%$ at baseline. The number of patients indicating to have an episode of nocturnal hypoglycemia remained unchanged after 3 months (29\%) but significantly decreased to $22 \%$ after 6 months $(\mathrm{p}<0.044)$. The number of patients indicating to have an episode of severe hypoglycemia, $8 \%,(\mathrm{~N}=7)$ did not change during the follow-up period.

The results regarding the changes of PRO are shown in Figure 1 and in Table 2. The DSC-r score decreases significantly from $17 \pm 14$ at baseline to $15 \pm 13$ after 3 months of follow-up ( $\mathrm{p}=0.016)$. This decrease is continued during the second observation period to $12 \pm 12$ after 6 months ( $p<0.001$ vs. baseline). The data on the DSC-r subscales are shown in Table 2. At baseline the subscale "fatigue" is particularly being indicated to be a diabetes related symptom that negatively influences wellbeing. This subscale significantly improves after 3 months and remains improved until the end of the observation period (Table 2).

The baseline score for the WHO-5 was $51 \pm 24$ and

Table 1. Baseline population characteristics and changes in clinical parameters.

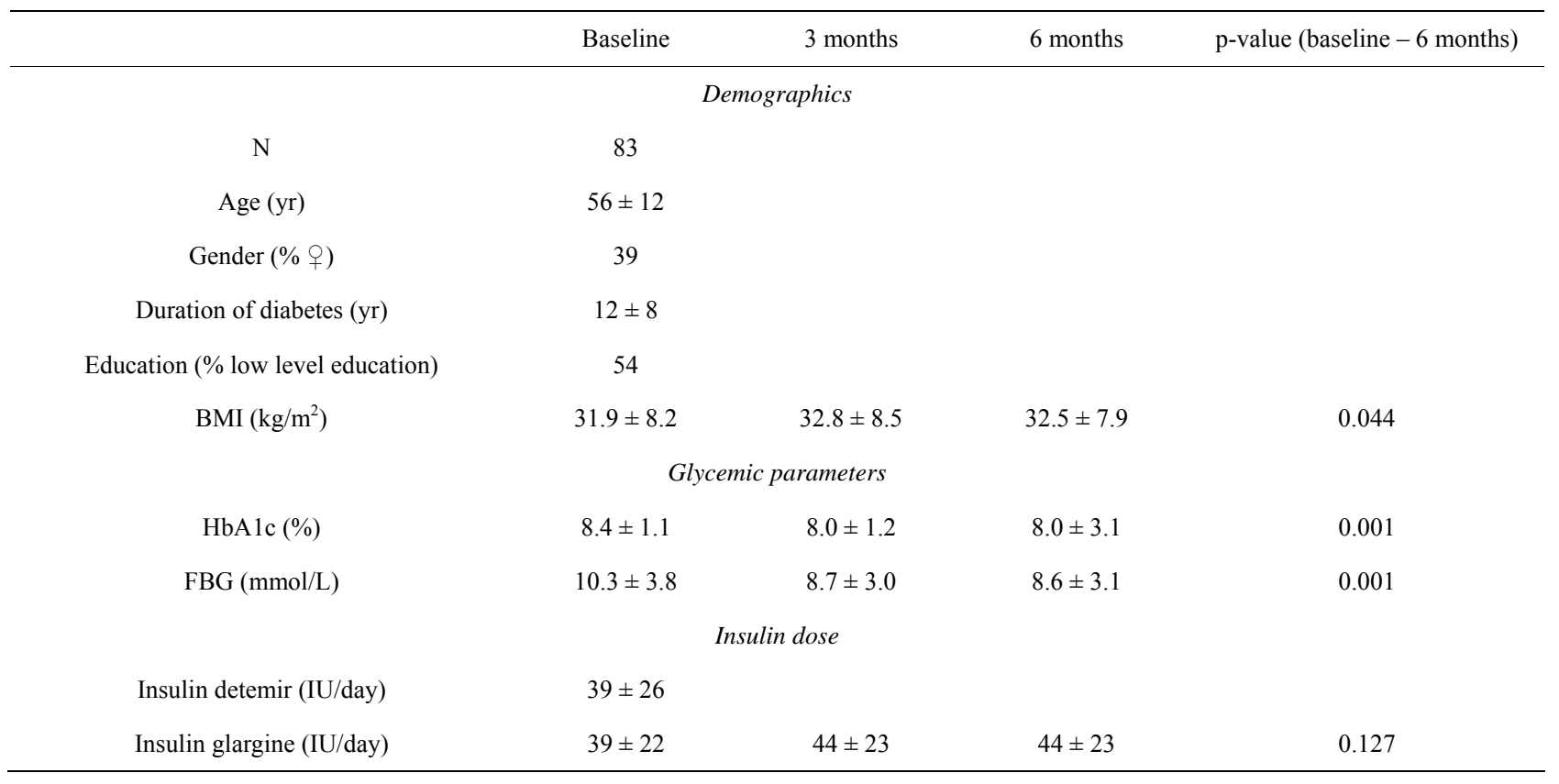




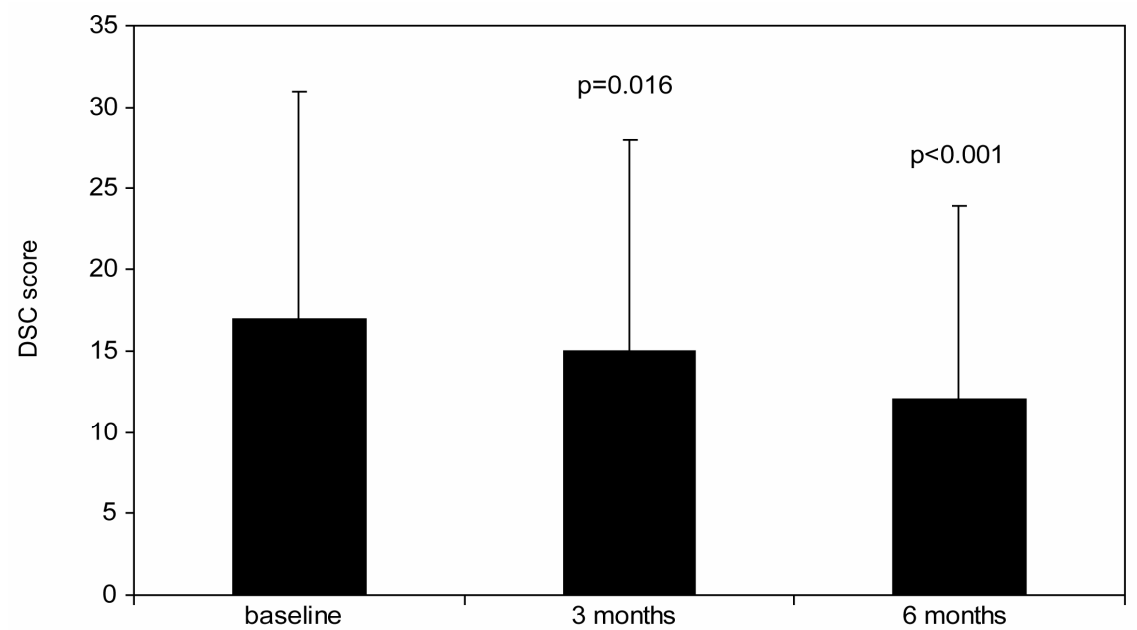

(a)

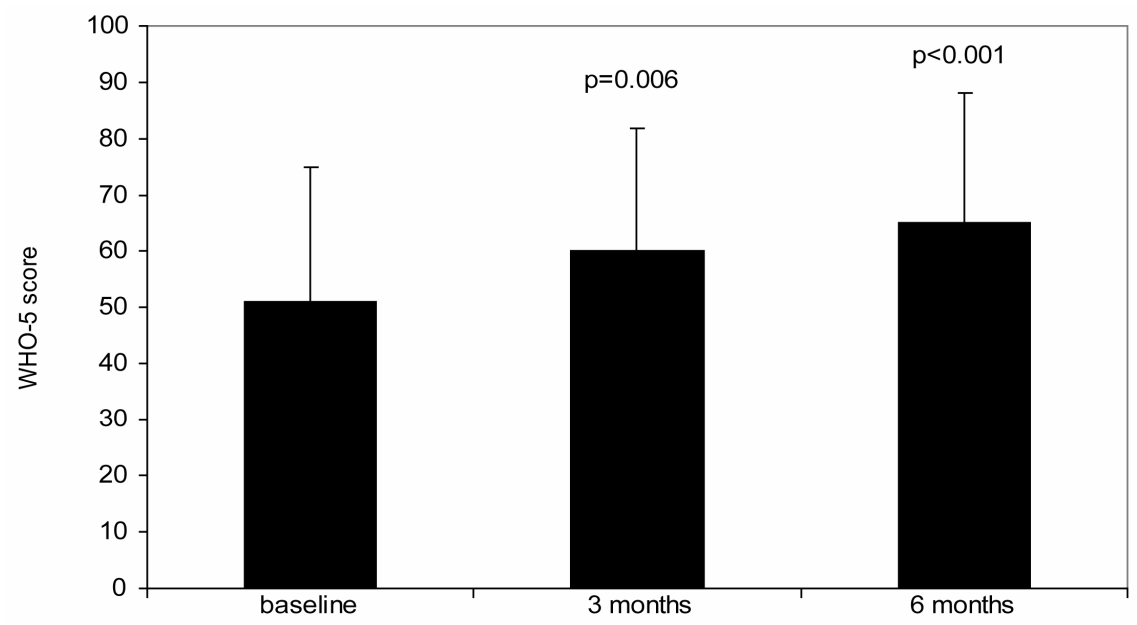

(b)

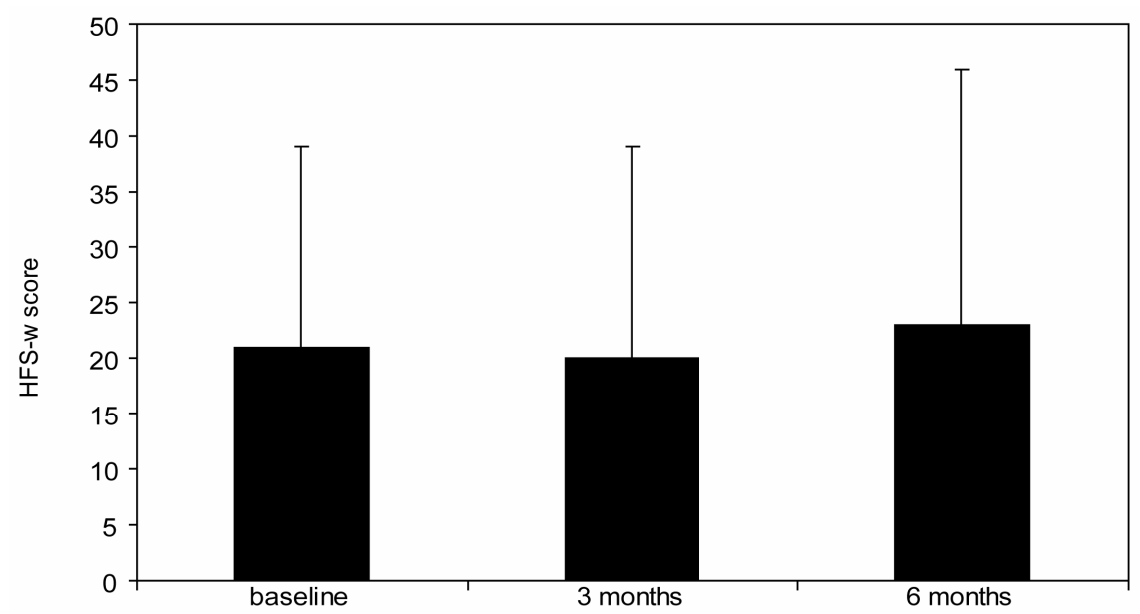

(c)

Figure 1. Change in PRO van from baseline to 6 months follow-up. (a): Diabetes Symptom Checklist-revised (DSC-r, scale 0 - 100, lower score indicates less complaints, (b): WHO-5 wellbeing score (WHO-5, scale 0 - 100, higher score indicates better wellbeing), (c): Hypoglycemia Fear Survey (HFS-w, scale 0 - 100, higher score indicates less fear for hypoglycemia). P-value represents significance compared to baseline value. 
Table 2. Change in DSC-r subscales over time.

\begin{tabular}{lcccc}
\hline & Baseline & 3 months & 6 months & p-value (baseline -6 months) \\
\hline DSC-r Total & $17 \pm 14$ & $15 \pm 13$ & $12 \pm 12$ & $<0.001$ \\
& & DSC-r subschales & & $<0.001$ \\
- Hyperglycemia & $21.5 \pm 22.0$ & $15.4 \pm 18.2$ & $11.4 \pm 14.5$ & $<0.001$ \\
- Hypoglycemia & $16.8 \pm 19.4$ & $14.1 \pm 15.5$ & $9.3 \pm 13.7$ & 0.001 \\
- Cognition & $18.0 \pm 20.1$ & $14.4 \pm 17.4$ & $11.8 \pm 14.7$ & 0.001 \\
- Fatigue & $33.9 \pm 27.0$ & $26.3 \pm 25.9$ & $22.8 \pm 24.4$ & 0.009 \\
- Cardiovascular & $13.3 \pm 16.0$ & $11.2 \pm 14.8$ & $8.6 \pm 11.8$ & 0.312 \\
- Neuropathy & $14.2 \pm 19.4$ & $13.0 \pm 18.4$ & $12.3 \pm 16.6$ & 0.314 \\
- Neuropathic pain & $12.1 \pm 19.3$ & $14.4 \pm 19.4$ & $14.3 \pm 30.3$ & 0.023 \\
- Retinopathy & $11.9 \pm 16.9$ & $9.2 \pm 16.2$ & $8.5 \pm 14.9$ & \\
\hline
\end{tabular}

improved to $60 \pm 22$ after 3 months and to $65 \pm 23$ after 6 months ( $\mathrm{p}<0.001 \mathrm{vs}$. baseline). Even after correction for glycemic control, by means of HbAlc similar WHO-5 scores are found (data not shown). At the beginning of the observation period approximately half of the patients $(51 \%, \mathrm{~N}=42)$ has a suboptimal or even lower WHO-5 score. This percentage significantly improved to $22 \%$ (N $=18$ ) after 6 weeks of treatment with insulin glargine. The HFS-w scores (worries about hypoglycemia) were low at baseline and remained unchanged during the remainder of the study.

\section{DISCUSSION}

The results of this 6-month, observational study show that conversion of the treatment from insulin detemir to insulin glargine in patients with DM2 as their basal insulin analogue induces a significant improvement in glycemic control and emotional wellbeing in Dutch daily practice.

International treatment guidelines, such as the ADA/ EASD consensus statement, suggest that the use of insulin-analogues with a longer duration of action and a peak-less pharmacologic profile, leads to a diminished prevalence of (nocturnal) hypoglycemia compared to $\mathrm{NPH}$-insulin [5]. A large number of studies confirm these findings [32-35]. Recently several head-to-head randomised clinical trials have compared both basal insulin-analogues, both in insulin-naïve patients and in patients using a basal-bolus insulin regimen [36-39]. In addition to the separate study results a recent Cochrane review shows an equal low number of hypoglycemic events and no clinically relevant difference in glycemic control between once daily insulin glargine and once to twice or twice daily insulin detemir [36-40]. There are, however, differences with respect to other, clinically relevant, secondary endpoints such as insulin dosage and dosing frequency and weight gain. In patients with DM2 using either basal insulin analogue in addition to oral medication up to $75 \%$ higher doses of insulin detemir are needed compared to insulin glargine to reach similar levels of glycemic control [36,37,40,41]. In addition, dosing frequency of insulin glargine appeared to be lower compared to insulin detemir, whereas weight gain was lower for insulin detemir [36-40]. Although it can be argued that a lower dosing frequency has a positive influence on treatment satisfaction and (hence) treatment adherence [42], the same can be argued for lower weight gain. Data from the L2T3 study suggest that treatment with once daily insulin glargine result in better patient satisfaction compared to treatment with twice daily insulin detemir, despite a difference of $0.8 \mathrm{~kg}$ in weight gain [37].

In the present study both the DSC-r score as well as the emotional wellbeing (WHO-5) show significant improvements, despite a small increase in BMI during the 6 months observation period. A similar observational study in the Netherlands in insulin-naïve patients starting insulin glargine treatment also showed that modest weight gain following insulin glargine initiation in DM2 was not associated with deterioration of HRQoL [43].

The average improvement of the WHO-5 score was 14 points, which is more than, according to the authors of the WHO-5 questionnaire, is considered to be clinically relevant [44]. Based on the DSC-r subscales it can be concluded that especially the scales associated with "fatigue" and "hyperglycemia" comprise the problems associated with a decrease in wellbeing. Indeed these are also the scales that show a significant improvement, in contrast to the robust subscale "neuropathic pain". Fear for hypoglycemia does not diminish after 6 months which is in accordance with the finding that the number of hypo- 
glycemic episodes does not change after 6 months.

An advantage of the observational character of the current study is that the results obtained in a strict controlled setting and in a selected patient population such as in a randomised controlled trial, are derived from daily reallife clinical practice. Simultaneously this advantage implies a disadvantage: the observational setting can cause selection bias. However, several arguments suggest that there was no such bias in this study. First, baseline $\mathrm{HbA} 1 \mathrm{c}$ was $8.4 \%$ suggesting that there was a good medical indication for the included patient population to change basal insulin therapy. Second, socio-economic patient characteristics show that the study population is representative for a patient population in normal clinical practice. Third, the improvements regarding the $\mathrm{HbAlc}$ and WHO-5 score is better than can be expected on the base of a so-called "study-effect" [44]. Apart from the study-effect the shown improvements in $\mathrm{HbAlc}$ and emotional wellbeing could partially be explained by optimisation of the insulin titration. Although it can not be excluded that dose optimisation has to some extent added to the positive study results, it is also likely that (part of) the positive effect is explained by the change of the insulin regimen and subsequent improvement in glycemic control. This is corroborated by the fact that only during the first 3 months a small increase in insulin dose was found with a concomitant improvement in $\mathrm{HbAlc}$ percentage, whereas the wellbeing continued to improve after these first 3 months, represented by a significant improvement of the WHO-5 and DCS-r scores from months 3 to 6 . Finally, even after correction for HbAlc of the WHO-5 score, wellbeing appeared to improve significantly.

In conclusion the results of this observational study show that changing basal insulin therapy to insulin glargine in patients with DM2 who are in poor glycemic control with insulin detemir leads to a clinically significant improvement of glycemic control as well as emotional wellbeing, despite a small increase in weight. These results should be confirmed in future (randomized comparative) studies.

\section{ACKNOWLEDGEMENTS}

The results of this study are based on a sub study of the ESPRIT project, funded by sanofi-aventis Netherlands [25]. Data-analysis was performed by T. Hajos, MSc at the Free University Medical Centre.

Financial support was given by sanofi-aventis Netherlands.

\section{REFERENCES}

[1] Heine, R.J., et al. (2006) Management of hyperglycaemia in type 2 diabetes. British Medical Journal, 333, 12001204. doi:10.1136/bmj.39022.462546.80
[2] Cook, M.N., et al. (2005) Glycemic control continues to deteriorate after sulfonylureas are added to metformin among patients with type 2 diabetes. Diabetes Care, 28, 995-1000. doi:10.2337/diacare.28.5.995

[3] Holman, R., et al. (2008) Intensive glucose control in type 2 diabetes. New England Journal of Medicine, 359, 1577-1589. doi:10.1056/NEJMoa0806470

[4] Rutten, G.E.H.M., et al. (2006) NHG-standaard diabetes mellitus type 2. Huisarts en Wetenschap, 49, 137-152.

[5] Nathan, D., et al. (2009) Management of hyperglycemia in type 2 diabetes: A consensus algorithm for the initiation and adjustment of therapy. Diabetologia, 52, 17-30. doi:10.1007/s00125-008-1157-y

[6] Rubino, A., et al. (2007) Delayed initiation of subcutaneous insulin therapy after failure of oral glucose-lowering agents in patients with type 2 diabetes: A populationbased analysis in the UK. Diabetic Medicine, 24, 1412 1418. doi:10.1111/j.1464-5491.2007.02279.x

[7] Cook, M., et al. (2007) Initial monotherapy with either metformin or sulphonylureas often fails to achieve maintain current glycaemic goals in patients with type 2 diabetes in UK primary care. Diabetic Medicine, 24, 350358. doi:10.1111/j.1464-5491.2007.02078.x

[8] Korytkowski, M. (2002) When oral agents fail: Practical barriers to starting insulin. International Journal of Obesity, 26, S18-S24. doi:10.1038/sj.ijo.0802173

[9] Peyrot, M., et al. (2005) Resistance to insulin therapy among patients and providers: Results of the cross-sectional Diabetes Attitudes, Wishes, and Needs (DAWN) study. Diabetes Care, 28, 2673-2679. doi:10.2337/diacare.28.11.2673

[10] Tabaei, B.P., et al. (2004) Glycemia and the quality of well-being in patients with diabetes. Quality of Life Research, 13, 1153-1161. doi:10.1023/B:QURE.0000031336.81580.52

[11] Rubin, R. (2005) Adherence to pharmacologic therapy in patients with type 2 diabetes mellitus. American Journal of Medicine, 118, 27S-24S.

doi:10.1016/j.amjmed.2005.04.012

[12] Lundkvist, J., et al. (2005) The economic and quality of life impact of hypoglycemia. European Journal of Health Economics, 6, 197-202.

[13] Cryer, P.E. and Childs, B.P. (2002) Negotiating the barrier of hypoglycemia in diabetes. Diabetes Spectrum, 15, 20-27. doi:10.2337/diaspect.15.1.20

[14] Polonsky, W., et al. (2005) Psychological insulin resistance in patients with type 2 diabetes: The scope of the problem. Diabetes Care, 28, 2543-2545. doi:10.2337/diacare.28.10.2543

[15] Wilson, M., et al. (2004) Treatment satisfaction after commencement of insulin in type 2 diabetes. Diabetes Research and Clinical Practice, 66, 263-267. doi:10.1016/j.diabres.2004.04.010

[16] Houlden, R., et al. (2007) Treatment satisfaction and quality of life using an early insulinization strategy with insulin glargine compared to an adjusted oral therapy in the management of type 2 diabetes: The Canadian INSIGHT study. Diabetes Research and Clinical Practice, 


\section{8, 254-258. doi:10.1016/j.diabres.2007.03.021}

[17] Vinik, A., et al. (2007) Adding insulin glargine versus rosiglitazone. Health-related quality of life impact in type 2 diabetes. Diabetes Care, 30, 795-800. doi:10.2337/dc06-1712

[18] Hajos, T., et al. (2011) Initiation of insulin glargine in patients with type 2 diabetes in suboptimal glycaemic control positively impacts health-related quality of life. A prospective cohort study in primary care. Diabetic Medicine, 28, 1096-1102.

doi:10.1111/j.1464-5491.2011.03329.x

[19] De Grauw, W.J.C., et al. (2001) Insulin therapy in poorly controlled type 2 diabetic patients: Does it affect quality of life? British Journal of General Practice, 51, 527-532.

[20] De Sonnaville, J.J., et al. (1998) Well-being and symptoms in relation to insulin therapy in type 2 diabetes. Diabetes Care, 21, 919-924. doi:10.2337/diacare.21.6.919

[21] Redekop, W., et al. (2002) Health-related quality of life and treament satisfaction in Dutch patients with type 2 diabetes. Diabetes Care, 25, 458-463. doi: $10.2337 /$ diacare.25.3.458

[22] Goddijn, P., et al. (1999) Longitudinal study on glycaemic control and quality of life in patients with type 2 diabetes mellitus referred for intensified control. Diabetes Medicine, 16, 23-30. doi:10.1046/j.1464-5491.1999.00002.x

[23] Davis, T., et al. (2001) Effect of insulin therapy on quality of life in type 2 diabetes mellitus: The fremantle diabetes study. Diabetes Research and Clinical Practice, 52, 63-71. doi:10.1016/S0168-8227(00)00245-X

[24] Horvath, K., et al. (2007) Long-acting insulin analogues versus NPH insulin (human isophane insulin) for type 2 diabetes mellitus. Cochrane Database of Systematic Reviews, 2, CD005613.

[25] Hajos, T., et al. (2009) Switching from NPH to insulin glargine positively impacts on glycemic control and psychological well-being in suboptimally controlled type 2 diabetes patients. An observational study. Diabetes, 58, A537

[26] Snoek, F.J., et al. (1996) The Dutch version of the hypoglycemia fear survey: Internal consistency and validity. Gedrag en Gezondheid, 24, 287-292.

[27] Grootenhuis, P., et al. (1994) Development of a type 2 diabetes symptom checklist: A measure of symptom severity. Diabetic Medicine, 11, 253-261. doi:10.1111/j.1464-5491.1994.tb00268.x

[28] Arbuckle, R., et al. (2009) Psychometric evaluation of the Diabetes Symptom Checklist-Revised (DSC-R)-A Measure of Symptom Distress. Value Health, 12, 1168-1175. doi:10.1111/j.1524-4733.2009.00571.x

[29] Bech, P., et al. (1996) The WHO (Ten) Well-being index: Validation in diabetes. Psychotherapy and Psychosomatics, 65, 183-190. doi:10.1159/000289073

[30] Lowe, B., et al. (2004) Comparative validity of three screening questionnaires for DSM-IV depressive disorders and physicians' diagnoses. Journal of Affective Disorders, 78, 131-140.

\section{doi:10.1016/S0165-0327(02)00237-9}

[31] Rubin, D.B. (1987) Multiple imputation for nonresponse in surveys. John Wiley \& Sons, New York. doi:10.1002/9780470316696

[32] Rosenstock, J., et al. (2005) Reduced hypoglycemia risk with insulin glargine. A meta-analysis comparing insulin glargine with human NPH insulin in type 2 diabetes. Diabetes Care, 28, 950-955.

doi:10.2337/diacare.28.4.950

[33] Mullins, P., et al. (2007) Negative binomial meta-regression analysis of combined glycosylated hemoglobin and hypoglycemia outcomes across eleven phase III and IV studies of insulin glargine compared with Neutral Protamine Hagendorn insulin in type 1 and type 2 diabetes mellitus. Clinical Therapeutics, 29, 1607-1619. doi:10.1016/j.clinthera.2007.08.020

[34] Hermansen, K., et al. (2006) A 26-week, randomized, parallel, treat-to-target trial comparing insulin detemir with NPH insulin as add-on therapy to oral glucose-lowering drugs in insulin-naïve people with type 2 diabetes. Diabetes Care, 29, 1269-1274. doi: $10.2337 / \mathrm{dc} 05-1365$

[35] Rossetti, P., et al. (2008) Superiority of insulin analogues versus human insulin in the treatment of diabetes mellitus. Archives of Physiology and Biochemistry, 114, 3-10. doi:10.1080/13813450801900777

[36] Rosenstock, J., et al. (2008) A randomised, 52 week, treat-to-target trial comparing insulin detemir with insulin glargine when administered as add-on to glucose-lowering drugs in insulin-naïve people with type 2 diabetes. Diabetologia, 51, 408-416. doi:10.1007/s00125-007-0911-x

[37] Swinnen, S., et al. (2010) A 24-week, randomized, treatto-target trial comparing initiation of insulin glargine once-daily with insulin detemir twice-daily in patients with type 2 diabetes inadequately controlled in oral glucose-lowering drugs. Diabetes Care, Publish ahead of Print, Published Online 3 March 2010.

[38] Hollander, P., et al. (2008) A 52-week, multinational, open-label, parallel-group, noninferiority, treat-to-target trial comparing insulin detemir with insulin glargine in a basal-bolus regimen with mealtime insulin aspart in patients with type 2 diabetes. Clinical Therapeutics, 30, 1976-1987. doi:10.1016/j.clinthera.2008.11.001

[39] Raskin, P., et al. (2009) Comparison of insulin detemir and insulin glargine using a basal-bolus regimen in a randomized, controlled clinical study in patients with type 2 diabetes. Diabetes/Metabolism Research and Reviews, 25, 542-548. doi:10.1002/dmrr.989

[40] Swinnen, S., et al. (2011) Insulin detemir versus insulin glargine for type 2 diabetes mellitus. Cochrane Database of Systematic Reviews, 7, CD006383. doi:10.1002/14651858.CD006383.pub2

[41] Swinnen, S., et al. (2009) Higher dose requirements with insulin detemir in type 2 diabetes-Three cases and a review of the literature. Diabetes Research and Clinical Practice, 84, e24-e26. doi:10.1016/j.diabres.2009.02.009

[42] Morris, A.D. (2003) Addressing dosing frequency in dia- 
betes: A simple approach to improving adherence to therapy and clinical outcomes. Diabetes Educator, 29, 440- 446. doi:10.1177/014572170302900309

[43] Hajos, T., et al. (2011) Modest weight gain following insulin glargine initiation is not associated with decreased health-related quality of life (HRQoL) in type 2 diabetes. Diabetes, 60, A229.
[44] Bech, P., Olsen, L.R., Kjoller, M. and Rasmussen, N.K. (2003) Measuring well-being rather than the absence of distress symptoms: A comparison of the SF-36 mental health subscale and the WHO-five well-being scale. International Journal of Methods in Psychiatric Research, 12, 85-91. doi: $10.1002 / \mathrm{mpr} .145$ 